\section{Cup-to-disc ratio: agreement between slit-lamp indirect ophthalmoscopic estimation and stratus optical coherence tomography measurement}

F Arnalich-Montiel, FJ Muñoz-Negrete,

G Rebolleda, M Sales-Sanz and C Cabarga shows higher values than the specialists; the greatest differences occurred when assessing small CDRs and the differences diminished as the cupping increased. These two methods of measurement are not interchangeable, and the difference must be considered, especially in discs with smaller CDRs.

Eye (2007) 21, 1041-1049; doi:10.1038/sj.eye.6702391; published online 5 May 2006

\section{Introduction}

Previous studies have shown that detectable visual field changes in glaucoma become evident after a significant neuronal loss occurs. ${ }^{1-3}$ These data suggest that early detection of glaucoma may be only achieved with technology that provides qualitative or quantitative measurements of the axons (nerve fibers) or the bodies of the retinal ganglion cells (RGCs), the main target in glaucomatous optic neuropathy. Quantitative changes in the thickness of the peripapillary retinal nerve fiber layer (RNFL), the morphology of the optic nerve head $(\mathrm{ONH})$, or both can be measured with several instruments such as confocal scanning laser ophthalmoscopy, scanning laser polarimetry, and optical coherence tomography (OCT). ${ }^{4-6}$ Macular RGCs are thought to be another target for scanning to detect early glaucomatous damage; however, in the present time it fails to detect early glaucomatous changes. ${ }^{7,8}$
Department of Ophthalmology, Hospital Ramón y Cajal, Glaucoma Unit, Madrid, Spain

Correspondence: Dr F Arnalich-Montiel, Department of Ophthalmology, Hospital Ramón y Cajal, Glaucoma Unit, Madrid, Spain Tel: + 34619608973 E-mail: arnalich@hotmail.com

Received: 30 November 2005

Accepted in revised form: 18 March 2006 Published online: 5 May 2006

The authors have no financial interest in the materials mentioned in this article 
Ophthalmoscopic estimation of the vertical cup-to-disc ratio (VCDR) of the optic nerve head is important in the management and follow-up of patients with glaucoma or glaucoma suspects, and has been found to correlate with global visual field indices. ${ }^{9}$ In a clinical setting, fundus biomicroscopy is frequently used for this, but it has only a moderate interobserver agreement and relies on observer experience. ${ }^{10,11}$

OCT is capable of measuring in a more reproducible way, a greater number of parameters than ophthalmoscopy.

OCT, a high-resolution noncontact imaging modality with great acceptance among ophthalmologists, is an excellent diagnostic tool for objective and quantitative measurement of ONH and RNFL parameters. ${ }^{7,8,12-14}$ The reproducibility of $\mathrm{ONH}$ measurements using the Stratus OCT (Carl Zeiss Meditec, Dublin, CA, USA) was reported recently to be sufficient, especially regarding the cup-to-disc ratios (CDRs). ${ }^{15}$ Although OCT may give objective measurement with less variation than biomicroscopy, this in itself does not mean that it will be more accurate in estimating the actual disc measurements.

As follow-up and examining change at higher CDR is a problem when using subjective measurements, a study of the relationship between CDR determined by OCT and indirect ophthalmoscopy seems necessary.

\section{Materials and methods}

\section{Patients}

A total of 81 consecutive patients (81 eyes) were recruited from the glaucoma service at Hospital Ramon y Cajal. All patients provided written informed consent. All procedures were performed according to the standards of the Declaration of Helsinki. The ethics committee of our institution approved the study protocol. This study included either patients with primary open-angle glaucoma (POAG) $(n=56)$ or ocular hypertension $(n=25)$. Table 1 shows the characteristics of the study population.

All participants underwent a complete ophthalmic study that included a detailed medical history, intraocular pressure (IOP) measurement using Goldmann applanation tonometry, gonioscopy, biomicroscopy through undilated and dilated pupils, and visual field testing using the Humphrey Field Analyzer II (Carl Zeiss Meditec, Dublin, CA, USA) 24-2 program with the Swedish Interactive Threshold Algorithm. The participants also underwent OCT ONH scanning (Carl Zeiss Meditec, Dublin, CA, USA). The refractive errors ranged from +3.00 to -6.00 diopters. Eyes were excluded if they had substantial media opacity or signs of posterior and/or optic nerve pathology other than those attributed to glaucoma. Patients were excluded if they had a tilted optic disc, which was reported recently to affect OCT measurements. ${ }^{16}$ When both eyes were eligible for the study, one eye was randomly selected, accounting for a total of 41 right eyes and 40 left eyes.

POAG was defined as an IOP consistently above $21 \mathrm{mmHg}$ without antiglaucoma treatment, an open drainage angle with no other possible explanation for the elevated IOP, and repeatable (three consecutive and reliable explorations) abnormal visual field test results (defined as glaucoma hemifield tests outside the normal limits or a pattern standard deviation outside the $95 \%$ normal confidence limits, regardless of the disc appearance). ${ }^{17,18}$ A reliable visual field test was defined

Table 1 Patients demographic characteristics

\begin{tabular}{|c|c|c|c|c|}
\hline & \multicolumn{2}{|c|}{ Glaucoma } & \multicolumn{2}{|c|}{ Ocular hypertension } \\
\hline Number of patients & \multicolumn{2}{|c|}{56} & \multicolumn{2}{|c|}{25} \\
\hline \multicolumn{5}{|l|}{ Sex } \\
\hline Female & \multirow{2}{*}{\multicolumn{2}{|c|}{$\begin{array}{l}33 \\
23\end{array}$}} & & \\
\hline \multirow[t]{2}{*}{ Male } & & & \multicolumn{2}{|c|}{13} \\
\hline & Mean & $\mathrm{SD}$ & Mean & SD \\
\hline Age (years) & 59 & 6.4 & 57 & 5.6 \\
\hline Disc area $\left(\mathrm{mm}^{2}\right)$ & 2.41 & 0.54 & 2.23 & 0.28 \\
\hline Disc diameter $(\mathrm{mm})$ & 1.82 & 0.28 & 1.80 & 0.28 \\
\hline Cup/disc horizontal ratio (OCT) & 0.69 & 0.18 & 0.58 & 0.20 \\
\hline Cup/disc horizontal ratio (glaucoma consultants) & 0.53 & 0.24 & 0.43 & 0.20 \\
\hline Cup/disc vertical ratio (OCT) & 0.66 & 0.18 & 0.56 & 0.19 \\
\hline Cup/disc vertical ratio (glaucoma consultants) & 0.58 & 0.24 & 0.45 & 0.20 \\
\hline
\end{tabular}

OCT, optical coherence tomography. 
as one with fewer than $20 \%$ fixation losses and falsepositive or false-negative responses. Ocular hypertension had the same criteria as for POAG but with normal visual fields.

\section{Testing}

Three glaucoma consultants with broad experience, who were masked to any clinical information, estimated during the same visit the VCDR and the horizontal cup-to-disc ratio (HCDR) to the nearest 0.05 increment between 0 (absence of cup disc) and 1.0 (absence of rim disc) by biomicroscopic indirect ophthalmoscopy using the Superfield lens (Volk Optical Inc., Mentor, OH, USA) after pupil dilation. The examiners' results were masked from the patients and each other. The examiners did not have access to the OCT results.

$\mathrm{ONH}$ scans of all patients were obtained during the same visit by an experienced examiner masked to the results of the other tests. Ocular imaging with dilated pupils used the currently available OCT, Stratus OCT. A scan was judged to be of good quality if the images of the ocular fundus were in focus, had a satisfactory signal-tonoise ratio $(>33 \mathrm{~dB})$, and the radial scans were centred on the optic disc. ${ }^{8}$ Whenever a patient did not meet these OCT criteria, the case was excluded from the study.

The Fast Optical Disc scanning protocol, which aligns and acquires automatically all the scans after the initial positioning by the operator, was used to obtain $\mathrm{ONH}$ measurements by existing Stratus OCT software (version 3.0). Disc margins are determined automatically as the end of the retinal pigment epithelium (RPE)/ choriocapillaris layer. A straight line connects the edges of the RPE/choriocapillaris, and a parallel line is constructed $150 \mu \mathrm{m}$ anteriorly. Structures below this line are defined as the disc cup and above this line as the neuroretinal rim. ${ }^{7}$ The parameters measured were the disc area, disc diameter, HCDR (defined as the ratio of the longest horizontal line across the cup to the longest horizontal line across the disc), and the VCDR (defined as the ratio of the longest vertical line across the cup to the longest vertical line across the disc).

\section{Statistical analysis}

The data were analysed using SPSS 12.0 for Windows (SPSS Inc., Chicago, IL, USA). The Student's $t$-test on paired observations was used to evaluate the differences in the VCDR and HCDR measurements between OCT and the glaucoma specialists and to evaluate differences between the right and left eyes. Intraclass correlation coefficients (ICCs), described by Shrout and Fleiss ${ }^{19}$ using the two-way mixed effects model, also were used to evaluate agreement. The measurement methods were considered to be fixed, whereas the people effects were random. As described by Bland and Altman, scatterplots of the differences between the two measurement methods against their averages were constructed to assess agreement. ${ }^{20}$ A regression coefficient $(r)$ of the average difference was calculated to estimate the proportional bias in the scatterplot.

\section{Results}

\section{Vertical cup-to-disc ratio}

The differences between the mean VCDR estimations obtained by each observer were not statistically significant. When considering the glaucoma specialists individually, there was a statistically significant difference between the mean Stratus OCT measurement and the mean ophthalmoscopic measurement of the VCDR. The mean OCT value was higher than the mean of the observers, with the difference ranging from 0.08 to 0.11 depending on the specialist (Table 2). When we divided the cases into three groups based on the average VCDR obtained by the specialists, the mean OCT value was higher for the VCDR less than $0.7(P<0.001)$. No differences were found for VCDRs of 0.7 or greater (Table 3). The power of this sample to detect differences above 0.05 was 0.92 .

A high level of agreement was found, when calculating the ICC for the VCDR among the three observers, which was 0.87 . When we evaluated the agreement between each observer and the OCT, the correlation also was high,

Table 2 Differences in VCDR estimation between specialists and OCT

\begin{tabular}{|c|c|c|c|c|c|}
\hline VCDR & Mean (SD) & $\begin{array}{c}\text { OCT VCDR } \\
\text { Mean difference }\end{array}$ & $\begin{array}{c}\text { OCT VCDR } \\
\text { 95\% Confidence interval }\end{array}$ & $\begin{array}{l}\text { OCT VCDR } \\
\text { P-value }\end{array}$ & $\begin{array}{c}\text { OCT VCDR } \\
\text { Intraclass correlation coefficient }\end{array}$ \\
\hline OCT & $0.63(0.19)$ & & & & \\
\hline Specialist 1 & $0.55(0.25)$ & 0.08 & $0.12-0.05$ & $<0.0001$ & 0.80 \\
\hline Specialist 2 & $0.52(0.25)$ & 0.11 & $0.14-0.08$ & $<0.0001$ & 0.82 \\
\hline Specialist 3 & $0.54(0.24)$ & 0.09 & $0.12-0.06$ & $<0.0001$ & 0.75 \\
\hline Mean of three specialists & $0.54(0.24)$ & & & & \\
\hline
\end{tabular}

VCDR, vertical cup-to-disc ratio; OCT, optical coherence tomography. 
Table 3 Mean differences between specialists and OCT based on the average VCDR measured by the specialists

\begin{tabular}{lccccc}
\hline & $\begin{array}{c}\text { OCT } \\
\text { mean }(S D)\end{array}$ & $\begin{array}{c}\text { Examiners } \\
\text { mean (SD) }\end{array}$ & $\begin{array}{c}\text { Mean difference (95\% } \\
\text { confidence interval) }\end{array}$ & No. of subjects & $\begin{array}{c}\text { P-value } \\
\text { (two-tailed t-test) }\end{array}$ \\
\hline VCDR 0-0.3 & $0.38(0.15)$ & $0.18(0.11)$ & $0.20(0.15-0.25)$ & 15 & $<0.001$ \\
VCDR 0.31-0.69 & $0.62(0.18)$ & $0.51(0.15)$ & $0.11(0.07-0.14)$ & 43 & $<0.001$ \\
VCDR 0.70-1 & $0.81(0.17)$ & $0.81(0.15)$ & $0(-0.03-0.03)$ & 23 & 0.98 \\
\hline
\end{tabular}

$\mathrm{OCT}$, optical coherence tomography; SD, standard deviation; $\mathrm{VCDR}$, vertical cup-to-disc ratio (average of observers values).

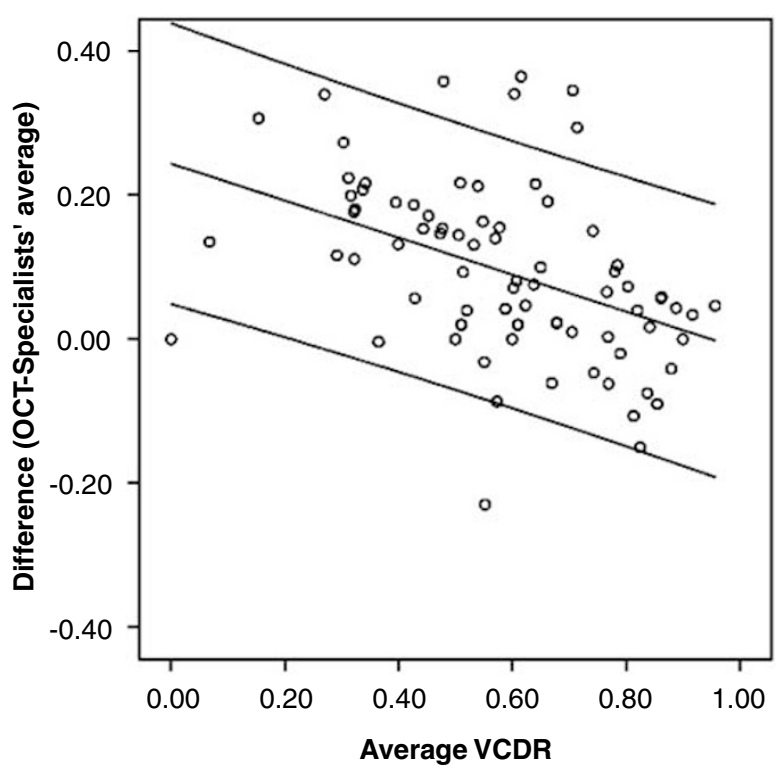

Figure 1 Bland-Altman plot comparing VCDR as measured by OCT and by ophthalmoscopy. Sample size of 81 patients. The existence of a proportional bias is shown by the significant slope of the line regressing the difference on the average $(r=-0.43$; $P<0.001)$. The regression line is shown with $95 \%$ individual confidence limits bands.

with ICCs ranging from 0.82 , the best result, to 0.75 , the lowest level of agreement (Table 2).

Figure 1 shows the agreement between the two methods in estimating the VCDR, where the difference (OCT VCDR minus the average VCDR of the specialists) is plotted against the average of the two measurements (OCT VCDR plus the average of the specialists VCDR/2). No significant systematic bias was observed, although a significant proportional bias was detected $(r=-0.43$; $P<0.001)$. For lower VCDR values, Stratus OCT tended to be much higher than the ophthalmoscopic values; for higher values, this difference decreased progressively toward 0 for average VCDRs above 0.7 .

The mean disc area was 2.35 (SD, 0.48). The median disc area was $2.29 \mathrm{~mm}^{2}$; 41 eyes had a disc area greater than $2.29 \mathrm{~mm}^{2}$, and 40 eyes had a disc area lesser than $2.29 \mathrm{~mm}^{2}$. In eyes with a disc area greater than $2.29 \mathrm{~mm}^{2}$, the mean VCDR was 0.66 (SD, 0.20) with OCT and 0.59

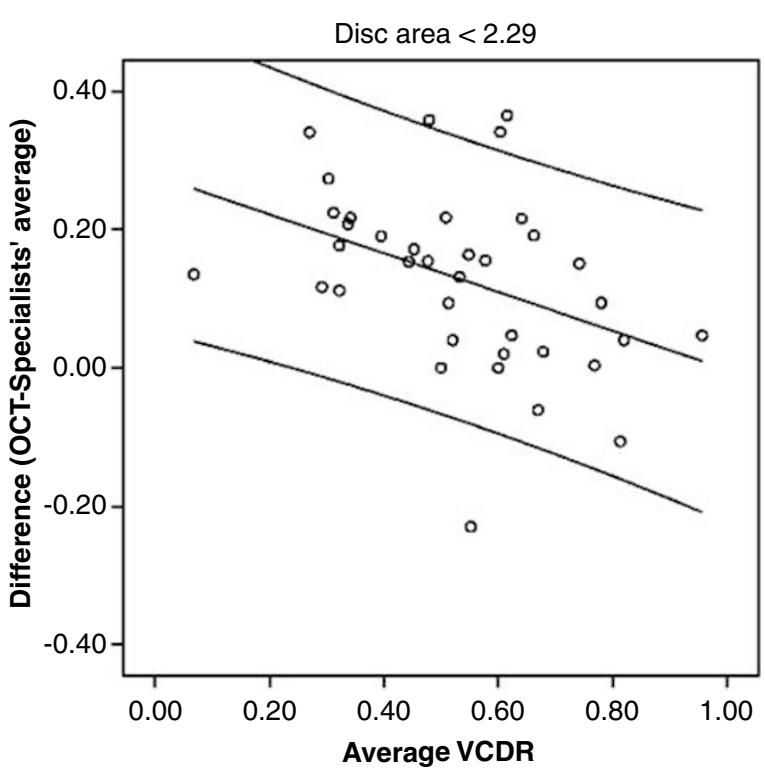

Figure 2 Bland-Altman plot comparing VCDR as measured by OCT and by ophthalmoscopy for smaller optic disc areas $\left(<2.29 \mathrm{~mm}^{2}\right)$. Sample size of 40 patients. The existence of a proportional bias is shown by the significant slope of the line regressing the difference on the average $(r=-0.41 ; P<0.01)$. The regression line is shown with $95 \%$ individual confidence limits bands.

(SD, 0.24) measured by the specialists. The difference of 0.07 was significant $(P<0.001)$. In eyes with a disc area less than $2.29 \mathrm{~mm}^{2}$, the mean VCDR was 0.60 (SD, 0.17) with OCT and $0.47(\mathrm{SD}, 0.22)$ measured by the specialists $(P<0.001)$. Figures 2 and 3 show similar slopes of the Bland-Altman plot when the disc areas were divided into two groups.

\section{Horizontal cup-to-disc ratio}

The mean OCT value for HCDR was statistically higher than that of the specialists. When considered separately, the differences ranged from 0.13 to 0.18 (Table 4). The mean HCDR values obtained by each observer indicated that the values measured by the third glaucoma specialist were significantly higher than those of the other two 
clinicians $(0.04, P<0.007$ and $0.05, P<0.001$ for clinicians 1 and 2, respectively).

When we divided the HCDR into three groups, the mean OCT HCDR values were greater in the three groups, with the differences ranging from $0.06(P=0.008)$

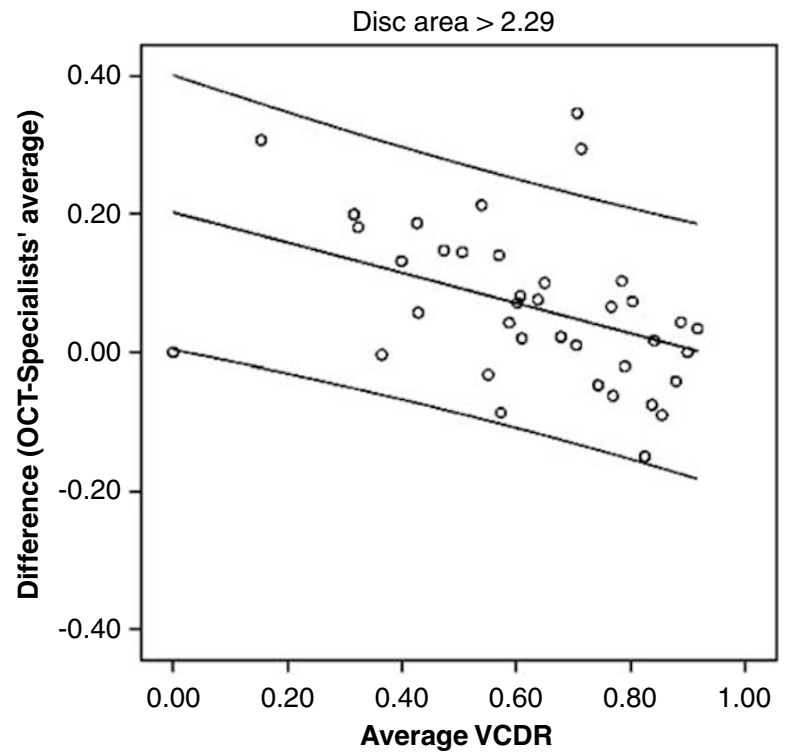

Figure 3 Bland-Altman plots comparing VCDR as measured by OCT and by ophthalmoscopy for larger optic disc areas $\left(>2.29 \mathrm{~mm}^{2}\right)$. Sample size of 41 patients. The existence of a proportional bias is shown by the significant slope of the line regressing the difference on the average $(r=-0.41 ; P<0.01)$. The regression line is shown with $95 \%$ individual confidence limits bands. for bigger cups to $0.25(P<0.001)$ for smaller cups (Table 5).

The ICC among the three observers was as high as 0.83 . The mean ICCs of each of the observers and OCT were 0.74 (Table 4 ).

The Bland-Altman plot (Figure 4) showed the presence of a proportional bias that reached statistical significance

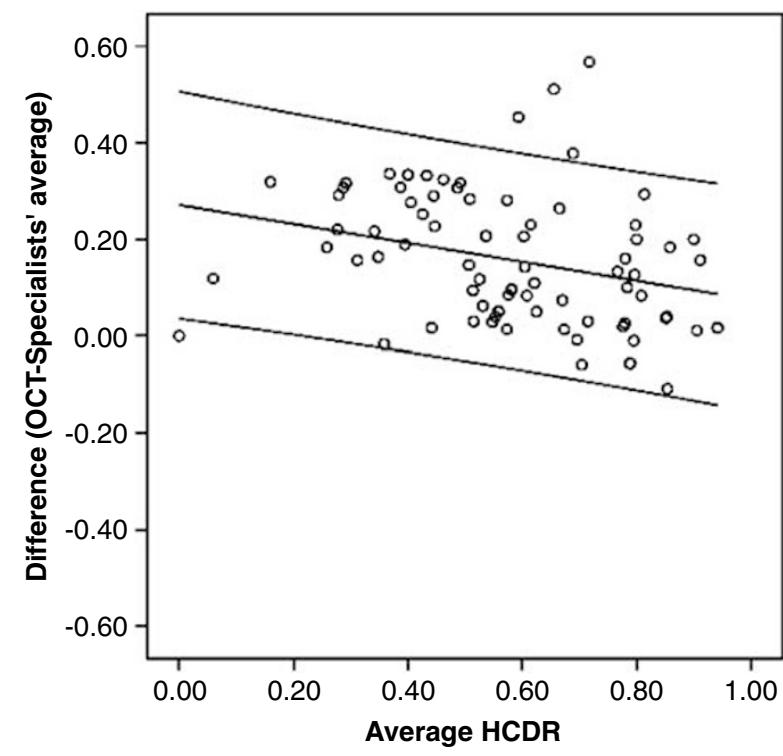

Figure 4 Bland-Altman plots comparing HCDR as measured by OCT and by ophthalmoscopy. Sample size of 81 patients. The existence of a proportional bias is shown by the significant slope of the line regressing the difference on the average $(r=-0.29$; $P<0.001$ ). The regression line is shown with $95 \%$ individual confidence limits bands.

Table 4 Differences in HCDR estimation between specialists and OCT

\begin{tabular}{|c|c|c|c|c|c|}
\hline$H C D R$ & Mean (SD) & $\begin{array}{c}\text { OCT HCDR Mean } \\
\text { difference }\end{array}$ & $\begin{array}{l}\text { OCT HCDR 95\% } \\
\text { confidence interval }\end{array}$ & $\begin{array}{c}\text { OCT HCDR } \\
\text { P-value }\end{array}$ & $\begin{array}{l}\text { OCT HCDR intraclass } \\
\text { correlation coefficient }\end{array}$ \\
\hline OCT & $0.66(0.20)$ & 0.16 & & & \\
\hline Specialist 1 & $0.49(0.25)$ & 0.17 & $0.20-0.13$ & $<0.0001$ & 0.74 \\
\hline Specialist 2 & $0.48(0.25)$ & 0.18 & $0.21-0.14$ & $<0.0001$ & 0.74 \\
\hline Specialist 3 & $0.53(0.24)$ & 0.13 & $0.16-0.09$ & $<0.0001$ & 0.74 \\
\hline Mean of three specialists & $0.50(0.23)$ & & & & \\
\hline
\end{tabular}

HCDR, horizontal cup-to-disc ratio; OCT, optical coherence tomography.

Table 5 Mean differences between specialists and OCT based on the average HCDR measured by the specialists

\begin{tabular}{lccccr}
\hline & OCT Mean (SD) & $\begin{array}{c}\text { Examiners } \\
\text { mean (SD) }\end{array}$ & $\begin{array}{c}\text { Mean difference } \\
\text { (95\% Confidence Interval) }\end{array}$ & No. of subjects & $\begin{array}{c}\text { P-value } \\
\text { (two-tailed t-test) }\end{array}$ \\
\hline HCDR 0-0.3 & $0.44(0.16)$ & $0.19(0.10)$ & $0.25(0.20-0.29)$ & 20 & $<0.001$ \\
HCDR 0.31-0.69 & $0.67(0.14)$ & $0.50(0.10)$ & $0.17(0.12-0.21)$ & 38 & $<0.001$ \\
HCDR 0.70-1 & $0.85(0.08)$ & $0.79(0.07)$ & $0.06(0.20-0.10)$ & 23 & 0.008 \\
\hline
\end{tabular}

OCT, optical coherence tomography; HCDR, horizontal cup-to-disc ratio (average of observers values). 


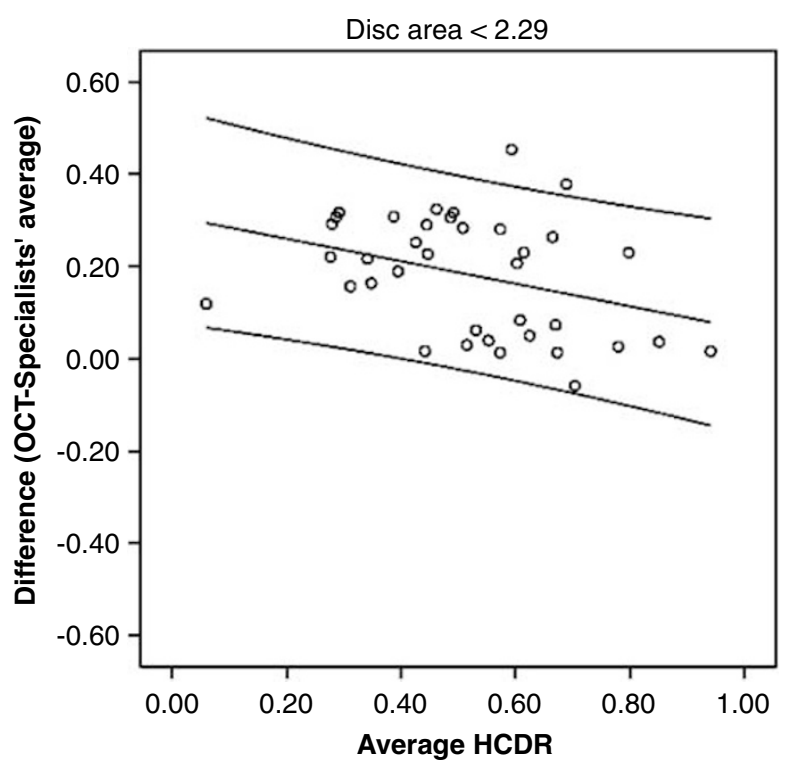

Figure 5 Bland-Altman plots comparing HCDR as measured by OCT and by ophthalmoscopy for smaller optic disc areas $\left(<2.29 \mathrm{~mm}^{2}\right)$. Sample size of 40 patients. The existence of a proportional bias is shown by the significant slope of the line regressing the difference on the average $(r=-0.34 ; P<0.05)$. The regression line is shown with $95 \%$ individual confidence limits bands.

( $r=-0.29$ for $P<0.001)$, with a greater difference for smaller degree of cupping.

In eyes with a disc area greater than $2.29 \mathrm{~mm}^{2}$, the mean OCT HCDR was 0.70 (SD, 0.22) and 0.55 (SD, 0.24) among the specialists. The difference of 0.15 was significant $(P<0.001)$. In eyes with a disc area less than $2.29 \mathrm{~mm}^{2}$, the mean OCT HCDR was 0.61 (SD, 0.17) and 0.43 (SD, 0.21 ) among the specialists $(P<0.001)$. The difference of 0.18 was statistically significant. Figures 5 and 6 show similar slopes of the Bland-Altman plot when the disc areas were divided into two groups, although when divided into two groups, the proportional bias lost significance for larger disc areas.

A comparison between the right and left eyes showed no differences in the HCDR or VCDR evaluation by OCT or the specialists.

\section{Discussion}

A number of recent studies have reported the ability of OCT to discriminate between healthy and glaucomatous eyes. ${ }^{5-8}$ Most of this research was based on the peripapillary thickness of the RNFL. New scanning areas for detecting glaucoma with OCT have been studied recently, especially the $\mathrm{ONH}$ and the macula. The former has shown highly discriminative indices for glaucoma, such as the VCDR or the cup-to-disc area ratio with a

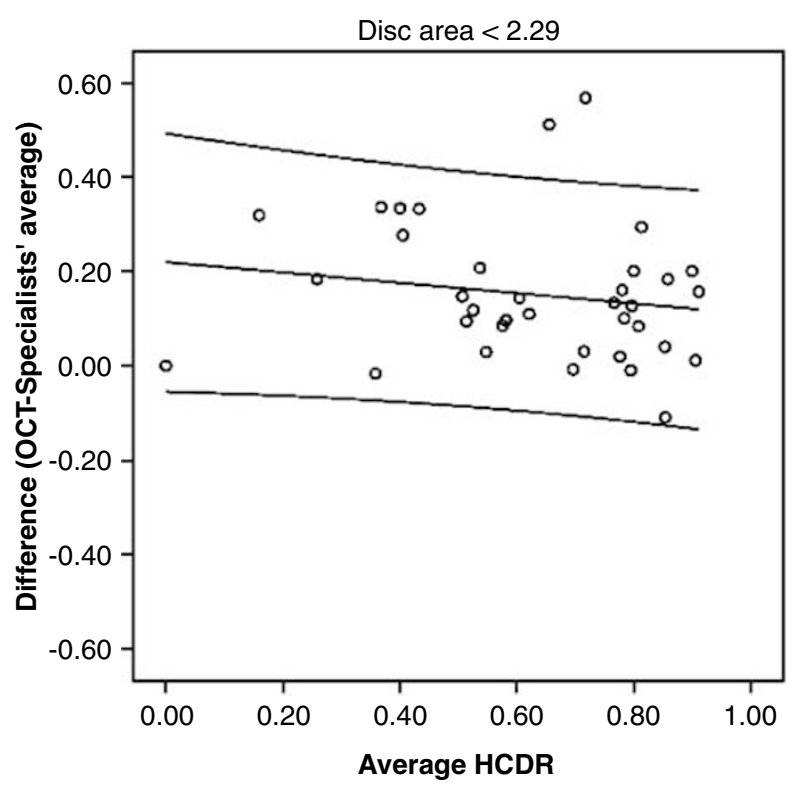

Figure 6 Bland-Altman plots comparing HCDR as measured by OCT and by ophthalmoscopy for larger optic disc areas $\left(>2.29 \mathrm{~mm}^{2}\right)$. Sample size of 41 patients. The existence of a proportional bias is shown by the significant slope of the line regressing the difference on the average $(r=-0.17 ; P=$ not significant). The regression line is shown with $95 \%$ individual confidence limits bands.

high power to discriminate between healthy and glaucomatous eyes. Clinical meaning of CDRs measured by OCT, and its relationship with the most common way of measuring with biomicroscopy needs to be studied.

Use of topographic evaluation of the ONH with OCT for glaucoma diagnosis still needs to be evaluated further. Stratus OCT ONH assessment provides objective measures of optic disc topography using an automated process of optic disc edge detection and cup delimitation based on the determination of the end of the RPE/ choriocapillaris layer. A manual algorithm for disc margin determination is also available. The manual and automatic algorithms perform comparably. ${ }^{14}$ To avoid introducing a subjective component into our analysis, we used the automatic algorithm. The automatic reference plane used for this study was $150 \mu \mathrm{m}$ above the RPE, which had the best performance for glaucoma detection and correlation with visual function when compared with other reference planes positioned at 95 and $205 \mu \mathrm{m}^{21}$

The purpose of the current study was to examine the relationship between the most common method of evaluating the $\mathrm{ONH}$ and the newly described OCT scanning area for glaucomatous damage based on $\mathrm{ONH}$ parameters.

We chose a slit-lamp indirect ophthalmoscopic determination of the CDR as the assessment method, 
because it provides a more accurate assessment of the CDR than direct ophthalmoscopy. ${ }^{22}$ Although previous studies have reported weak interobserver agreement of ophthalmoscopic assessment based on low ICC, we found a high ICC for both the VCDR and the HCDR. ${ }^{10,11}$ However, the agreement in the estimation of the HCDR was less strong, and one of the specialists had a value that was significantly higher. The oval shape of the normal optic disc, with a larger vertical than horizontal dimension, could justify, that estimating the HCDR could be more difficult and less accurate. Although the differences were significant, we considered only 0.040.05 as clinically irrelevant. However, it is well known that the correlation model measures show only the strength of a relationship between two variables and not their agreement. ${ }^{23}$ The great interobserver agreement that we found may be due to the extensive experience of the observers, because interobserver agreement increases with experience, and may not be the rule in standard clinical practice. ${ }^{24}$

Previous studies have compared other methods of quantitative measurements with qualitative measures. Watkins et $a l^{22}$ reported that direct ophthalmoscopy and fundus biomicroscopy were biased toward underestimation when compared with Heidelberg Retina Tomography (HRT), although the differences with biomicroscopy were smaller than those we found. Ikram et $a l^{25}$ compared ophthalmoscopy with other semiautomated systems of optic disc analysis and also found that there was ophthalmoscopic underestimation of the VCDR when compared with HRT and that the difference was even greater when compared with the ImageNet System. The possibility that the observers considered optic disc pallor and not the bending of the vessels as the beginning of cupping or included Elschnig's ring, as speculated by Ikram et al, ${ }^{25}$ is unlikely to have affected our results, as the observers were experienced glaucoma consultants. Correnti $\mathrm{el} \mathrm{al}^{26}$ found that subjective assessment also underestimates stereophotography.

None of the quantitative methods of assessment has been shown to perform better than qualitative assessment with stereoscopic colour photographs by experienced observers at distinguishing normal eyes from those with early to moderate glaucoma. ${ }^{5}$ Medeiros et $a l^{8}$ recently compared stereophotography assessment and OCT measurements. Although there was no difference in the mean VCDR of stereophotography and OCT, for lower values of VCDR, the OCT measurements were higher, whereas for greater VCDRs, the OCT measurements were lower. For the HCDR, Stratus OCT values were always higher in the same quantity, no matter what the value of the HCDR. ${ }^{8}$ These results are somewhat similar to those in the current study.
Hrynchak et $a l^{27}$ recently studied the relationship between biomicroscopy and OCT measurement of CDR. OCT cup widths were determined using two criteria: the first measured the separation of cup walls halfway down each side and the second estimated the separation $1 / 3$ down each side edge corresponding to one-half and one-third, the depth of the cup. For the former, the mean difference was centred around 0 , whereas for the latter, biomicroscopy underestimated the OCT measurements. That study included 20 healthy patients, and therefore, the level of concordance between clinical and morphometric estimates with OCT could not be established precisely for larger CDRs or patients with glaucoma. To the best of our knowledge, the current study is the first to include patients with either glaucoma or ocular hypertension. Because we have not studied normal subjects, we cannot establish whether the ophthalmoscopic underestimation found in this study for hypertensive and glaucomatous patients would be similar in normal degree of cupping.

Our results showed a highly significant ICC between the two methods of measuring HCDR and VCDR; however, when Bland and Altman plots were analysed, important disagreement was detected, probing once more the low utility of correlations coefficients to assess agreement between methods of measurements, an issue that has already been extensively acknowledged in the literature. ${ }^{20,23}$

There is not yet a normative scale for Stratus OCT ONH parameters. As shown in Figure 1, we can conclude that for VCDR, OCT tends to show higher values, and therefore the two measurement methods are not interchangeable. However, the difference decreases as the degree of cupping increases, showing good agreement with VCDR above 0.7. Normal eyes usually have less degree of cupping, therefore when evaluating smaller VCDR we likely will have to correct the OCT values. However, eyes at higher risk of glaucomatous optic neuropathy have an ophthalmoscopic VCDR above 0.7 ( 97.5 th percentile of the normal population). ${ }^{28}$ As previously stated, values above 0.7 are similar with either measurement method. In these cases, Stratus OCT $\mathrm{ONH}$ assessment provides more reliable and reproducible measures of optic disc topography for evaluating current status. Clinically, of course, one would wish to have better estimates of smaller VCDR to detect eyes with earlier glaucoma.

Regarding HCDR, OCT also provides higher values (with a greater difference than for the VCDR), and although the difference also diminishes as the degree of cupping increases, with values above 0.7 , agreement is still not complete. Therefore, when comparing ophthalmoscopy with OCT results, an adjustment must always be made. 
Zangwill $e t$ al $^{29}$ also found that the difference was larger in normal subjects (with smaller degree of cupping) than in patients with glaucoma when comparing stereophotography with HRT, suggesting that the reason for their findings was that clinicians often measure the cup diameter at the centre of the cup, whereas the HRT measures the diameter of the cup as it passes through the vertical meridian of the disc. Therefore, in glaucomatous eyes, a larger cup will have a wider area of the cup passing through the vertical meridian of the disc, and the difference between the two methods of estimation will be less.

When comparing the difference between large and small optic discs, we found that OCT overestimation of the cupping compared with observers is greater for smaller optic discs. The fact that increased CDRs are associated with larger ONHs is well known, ${ }^{30,31}$ and as we showed earlier, the higher the cupping the lower the difference. With larger ONHs and greater CDRs, the differences were therefore smaller.

In conclusion, we found good agreement between observers when evaluating the VCDR and HCDR with slit-lamp indirect ophthalmoscopy in patients with ocular hypertension and POAG. ONH analysis with OCT shows good agreement with slit-lamp indirect ophthalmoscopy for HDCR and VCDR evaluation in greater CDRs and disc areas. However, for smaller CDRs and disc areas, the OCT values tended to be higher.

\section{References}

1 Sommer A, Katz J, Quigley HA, Millar NR, Robin AL, Richter RC et al. Clinically detectable nerve fiber atrophy precedes the onset of glaucomatous field loss. Arch Ophthalmol 1991; 109: 77-83.

2 Zeyen TG, Caprioli J. Progression of disc and field damage in early glaucoma. Arch Ophthalmol 1993; 111: 62-65.

3 Harweth RS, Carter-Dawson L, Shen F, Smith III EL, Crawford ML. Ganglion cell losses underlying visual field defects from experimental glaucoma. Invest Ophthalmol Vis Sci 1999; 40: 2242-2250.

4 Mistlberger A, Liebmann JM, Greenfield DS, Hoh ST, Ishikawa $\mathrm{H}$, Marmor $\mathrm{M}$ et al. Assessment of optic disc anatomy and nerve fiber layer thickness in ocular hypertensive subjects with normal short wavelength automated perimetry. Ophthalmology 2002; 109: 1362-1366.

5 Greaney MJ, Hoffman DC, Garway-Heath DF, Nakla M, Coleman AL, Caprioli J. Comparison of optic nerve imaging methods to distinguish normal eyes from those with glaucoma. Invest Ophthalmol Vis Sci 2002; 43: 140-145.

6 Nouri-Mahdavi K, Hoffman BA, Tannenbaum DP, Law SK, Caprioli J. Identifying early glaucoma with optical coherence tomography. Am J Ophthalmol 2004; 137: 228-235.

7 Wollstein G, Ishikawa H, Wang J, Beaton SA, Schuman JS. Comparison of three optical coherence tomography scanning areas for detection of glaucomatous damage. Am J Ophthalmol 2005; 139: 39-43.
8 Medeiros FA, Zangwill LM, Bowd C. Evaluation of retinal nerve fiber layer, optic nerve head, and macular thickness measurements for glaucoma detection using optical coherence tomography. Am J Ophthalmol 2005; 139: 44-55.

9 Airaksinen PG, Drance SM, Douglas GR, Schulzer M. Neuroretinal rim areas and visual field indices in glaucoma. Am J Ophthalmol 1985; 99: 107-110.

10 Lichter PR. Variability of expert observers in evaluating the optic disc. Trans Am Ophthalmol Soc 1976; 74: 532-572.

11 Tielsch JM, Katz J, Quigley HA, Miller NR, Sommer A Intraobserver and interobserver agreement in measurements of optic disc characteristics. Ophthalmology 1988; 95: 350-356.

12 Schuman JS, Hee MR, Arya AV, Pedut-Kloizman T, Puliafito CA, Fujimoto JG et al. Optical coherence tomography: a new tool for glaucoma diagnosis. Curr Opin Ophthalmol 1995; 6: 89-95.

13 Schuman JS, Pedut-Kloizman T, Hertzmark E, Hee MR, Wilkins JR, Coker JG et al. Reproducibility of nerve fiber layer thickness measurements using optical coherence tomography. Ophthalmology 1996; 103: 1889-1898.

14 Schuman JS, Wollstein G, Farra T, Hertzmark E, Aydin A, Fujimoto JG et al. Comparison of optic nerve head measurements obtained by optical coherence tomography and confocal scanning laser ophthalmoscopy. Am J Ophthalmol 2003; 135: 504-512.

15 Paunescu LA, Schuman JS, Price LL, Stark PC, Beaton S, Ishikawa $\mathrm{H}$ et al. Reproducibility of nerve fiber thickness, macular thickness, and optic nerve head measurements using StratusOCT. Invest Ophthalmol Vis Sci 2004; 45: 1716-1724.

16 Park CY, Kim YT, Kee C. Evaluation of the influence of tilt optic disc on the measurement of optic disc variables obtained by optical coherence tomography and confocal scanning laser ophthalmoscopy. J Glaucoma 2005; 14: 210-214.

17 Hodapp E, Parrish II RK, Anderson DR. Clinical Decisions in Glaucoma. CV Mosby: St Louis, 1993, pp 46-47.

18 Katz J, Quigley HA, Sommer A. Detection of incident field loss using the glaucoma hemifield test. Ophthalmology 1996; 103: $657-663$.

19 Shrout PE, Fleiss JL. Intraclass correlations: uses in assessing rater reliability. Psychol Bull 1979; 86: 420-428.

20 Bland JM, Altman DG. Statistical methods for assessing agreement between two methods of clinical measurement. Lancet 1986; 1: 307-310.

21 Leung CK, Chan WM, Hui YL, Yung WH, Woo J, Tsang MK et al. Analysis of retinal nerve fiber layer and optic nerve head in glaucoma with different reference plane offsets, using optical coherence tomography. Invest Ophthalmol Vis Sci 2005; 46: 891-899.

22 Watkins R, Panchal L, Uddin J, Gunvant P. Vertical cup-todisc ratio: agreement between direct ophthalmoscopic estimation, fundus biomicroscopic estimation, and scanning laser ophthalmoscopic measurement. Ophthalmol Vis Sci 2003; 80: 454-459.

23 Cesana BM. Comparison of measurement methods requires appropriate statistical methodology. Am J Ophthalmol 2004; 137: 787-788.

24 Hanson S, Krishnan SK, Phillips J. Observer experience and cup:disc ratio assessment. Ophthalmol Vis Sci 2001; 78: 701-705.

25 Ikram MK, Borger PH, Assink JJ, Jonas JB, Hofman A, de Jong PT. Comparing ophthalmoscopy, slide viewing, and 
semiautomated systems in optic disc morphometry. Ophthalmology 2002; 109: 486-493.

26 Correnti AJ, Wollstein G, Price LL, Schuman JS. Comparison of optic nerve head assessment with digital stereoscopic camera (discam), scanning laser ophthalmoscopy and stereophotography. Ophthalmology 2003; 110: 1499-1505.

27 Hrynchak P, Hutchings N, Jones D, Simpson T. A comparison of cup to disc ratio measurement in normal subjects using optical coherence tomography image analysis of the optic nerve head and stereo fundus biomicroscopy. Ophthalmol Physiol Opt 2004; 24: 543-550.

28 Wolfs RC, Borger PH, Ramrattan RS, Klaver CC, Hulsman $\mathrm{CA}$, Hofman A et al. Changing views on open angle glaucoma: definitions and prevalences - The Rotterdam Study. Invest Ophthalmol Vis Sci 2000; 41: 3309-3321.

29 Zangwill L, Shakiba S, Caprioli J, Weinreb. Agreement between clinicians and a confocal scanning laser ophthalmoscope in estimating cup/disk ratios. Am J Ophthalmol 1995; 119: 415-421.

30 Briton RJ, Drance SM, Schulzer M, Douglas GR, Mawson DK. The area of the neuroretinal rim of the optic nerve in normal eyes. Am J Ophthalmol 1987; 103: 497-504.

31 Garway-Heath DF, Ruben ST, Viswanathan A, Hitchings RA. Vertical cup/disc ratio in relation to optic disc size: its value in the assessment of the glaucoma suspect. $\mathrm{Br} J$ Ophthalmol 1998; 82: 1118-1124. 